\title{
Crianças nos parques: segurança acima de tudo
}

| ${ }^{1}$ Simone Medeiros de Oliveira, ${ }^{2}$ Maria Paula Maia dos Santos, ${ }^{3}$ Paula Silva |

Resumo: Os parques públicos são espaços ao ar livre, que podem contribuir para o aumento da atividade física das crianças. $\mathrm{O}$ objetivo deste estudo, partindo da avaliação das características ambientais físicas dos parques, é identificar fatores relatados por crianças usuárias de parques que podem influenciar a sua frequência de utilização dos parques. Estudo descritivo e exploratório de abordagem qualitativa com a aplicação do instrumento EAPRS para avaliação da oferta estrutural dos parques e realização de grupos focais com crianças usuárias dos parques. Os resultados deste estudo indicam que a segurança percebida pelas crianças e a manutenção geral dos parques podem contribuir para a maior frequência desse público nos parques. Estratégias transdisciplinares visando a melhorias na segurança e na manutenção geral dos parques públicos são necessárias para promover a maior frequência infantil nesses locais.

> Palavras-chave: parques; crianças; atividade física.

\author{
1 Centro de Investigação em \\ Atividade Física, Saúde e \\ lazer (CIAFEL), Faculdade do \\ Desporto da Universidade do \\ Porto (FADEUP), Porto, Portugal \\ (simonemedeiros2005@hotmail. \\ com). \\ ORCID: 0000-0002-3700-4887 \\ ${ }^{2}$ Centro de Investigação em \\ Atividade Física, Saúde e \\ lazer (CIAFEL), Faculdade do \\ Desporto da Universidade do \\ Porto (FADEUP), Porto, Portugal \\ (msantos@fade.up.pt). \\ ORCID: 0000-0002-2182-9841 \\ ${ }^{3}$ Centro de Investigação em \\ Atividade Física, Saúde e \\ lazer (CIAFEL), Faculdade do \\ Desporto da Universidade do \\ Porto (FADEUP), Porto, Portugal \\ (psilva@fade.up.pt). \\ ORCID: 0000-0003-4231-9469
}

Recebido em: 05/08/2017 Aprovado em: 18/03/2018 Revisado em: 05/04/2018 


\section{Introdução}

Os parques são os ambientes naturais mais comuns nos meios urbanos e proporcionam aos indivíduos maior oportunidade de exposição à natureza, cada vez mais escassa nos centros urbanos (GLADWELL et al., 2013). A relação entre o ambiente natural e a saúde dos indivíduos é um tema em destaque no meio científico, que ressalta os benefícios que a frequência de ambientes naturais traz para a saúde mental, o bem-estar (ADINOLFI et al., 2014) e a vitalidade (VAN DEN BERG et al., 2016) dos indivíduos adultos. Esses ambientes também proporcionam melhoria na qualidade de vida das crianças (MCCRACKEN et al., 2016) e no seu bemestar emocional (WARD et al., 2016). Um estudo recente (RICHARDSON et al., 2017) enfatiza o papel facilitador de interaçóes socialmente benéficas dos ambientes naturais, especialmente nas crianças, uma vez que há uma maior oportunidade de interação entre pares e intercâmbio sociocultural.

Nesse cenário, atividade física (AF) é evidenciada no meio científico como um dos protagonistas (KACZYNSKI; HENDERSON, 2008; HARTIG et al., 2014), sendo definida como "qualquer movimento corporal produzido pelos músculos esqueléticos que resulta em gasto energético" (CASPERSEN et al., 1985). A prática da AF em ambientes naturais, ou ao ar livre, é reconhecida como uma das melhores estratégias de impacto positivo sobre a saúde dos indivíduos (GLADWELL et al., 2013; LEE et al., 2015).

Nas crianças, a AF está associada positivamente ao ambiente ao ar livre (TRAN et al., 2013; KLINKER et al., 2014a; SILVA; SANTOS, 2016), à presença de pares (FLOYD et al., 2011; MACDONALD-WALLIS et al., 2011; PEARCE et al., 2014), ao estilo de conduta permissivo maternal (JAGO et al., 2011), ao gênero masculino e à faixa etária das crianças (KLINKER et al., 2014b). Quando a AF é realizada em ambientes ao ar livre e fora do contexto escolar, apresenta associação positiva com a AF diária total (SCHOEPPE et al., 2014) e com os níveis de intensidade da AF praticada pelas crianças (KURKA et al., 2015). Particularmente nos parques, pela possibilidade de interaçáo entre pares ativos, pode contribuir para o maior envolvimento em AF moderada-vigorosa (FLOYD et al., 2011).

A oferta ampliada de parques é uma estratégia adotada pelo setor público para a promoção da saúde através da AF (LARSON et al., 2016). Todavia, sua utilização está associada à combinação entre suas características estruturais e as 
características individuais de seus usuários (BEDIMO-RUNG et al., 2005). A acessibilidade, a percepção de segurança, a qualidade, a limpeza e a tranquilidade são fatores associados à utilização dos ambientes naturais urbanos (LEE et al., 2015), verificando-se uma associação positiva entre o uso e a oferta de estruturas (BARAN et al., 2014). Entretanto, a quantidade de usuários dos parques pode estar relacionada com as dimensôes do parque e com a existência de atividades organizadas (COHEN et al., 2010). A percepção dos supervisores das crianças acerca da oferta de estruturas (BOHN-GOLDBAUM et al., 2013), a proximidade à residência das crianças e a densidade verde dos parques são fatores que podem favorecer a maior utilização dos parques pelas crianças (DUNTON et al., 2014).

As áreas próximas à residência das crianças revelam-se como potenciais ambientes promotores da sua AF (MITCHELL et al., 2016; PERRY et al., 2016). A percepção parental sobre os arredores da residência da criança pode ser um facilitador da AF moderada-vigorosa (KURKA et al., 2015) e da mobilidade independente das crianças (SANTOS et al., 2013). A presença de parques em zonas residenciais é associada a benefícios para a saúde através da AF (LARSON et al., 2016) e para o incremento dos níveis de intensidade da AF praticada pelos residentes locais (HAN et al., 2013). No entanto, a presença do parque não garante os benefícios para a saúde dos indivíduos (LEE et al., 2015) nem tampouco a utilização desse ambiente pelos residentes locais (COHEN et al., 2016). O fato de os parques proporcionarem oportunidades de comportamentos fisicamente ativos, como também sedentários, leva à necessidade de compreender as suas características ambientais e as possíveis associaçôes com os níveis de AF praticados nesses ambientes (BEDIMO-RUNG et al., 2005). No contexto português, a percentagem de crianças que afirmam brincar ao ar livre é superior às que referem praticar um desporto (SILVA; SANTOS, 2016), sendo importante estudar o contributo da AF desenvolvida pelas crianças nos ambientes naturais, como os parques.

Conhecer as características das estruturas dos parques, sua utilização e as percepçôes de seus usuários, particularmente as crianças, poderá contribuir para a maior utilização desses ambientes ao ar livre (COHEN et al., 2016) e para o aumento do comportamento fisicamente ativo das crianças. No presente estudo, pretende-se, partindo da avaliação das características ambientais físicas dos parques, identificar fatores relatados por crianças utilizadoras desses lugares que podem influenciar a sua frequência de utilização. 


\section{Métodos}

Este estudo de caráter descritivo-exploratório com uma abordagem qualitativa integra um projeto de doutorado em Atividade Física e Saúde, aprovado pelo Comitê de Ética da Faculdade do Desporto da Universidade do Porto, Portugal (processo CEFADE 07.2015). A amostra foi constituída por dois parques públicos (P1 e P2), com parque infantil, da cidade de Guimaráes (Portugal) e quatro grupos focais (GF), com crianças usuárias desses parques ( $\mathrm{n}=36$ crianças).

A avaliação da oferta das estruturas (instalaçôes e facilidades) dos parques foi realizada através do instrumento Environmental Assessment of Public Recreation Spaces (EAPRS) (Saelens et al., 2006), versão 8.0/2011, que consiste numa observação direta das estruturas e da qualidade da sua funcionalidade. As observaçóes seguiram a sequência proposta, resultando na pontuação dos 16 itens analisados. Em ambos os parques, a avaliação foi feita durante o outono, no período matinal, com duração média de 1 hora, não havendo remodelação dos parques durante a avaliação. Os resultados foram transcritos para um ficheiro Excel para cálculo da pontuação e usados para informaçóes descritivas sobre as características estruturais dos parques. A coleta, tabulação e análise dos dados foram realizadas pela mesma avaliadora.

Para identificação dos fatores associados à frequência de utilização dos parques pelas crianças foram realizados quatro GFs de nove crianças com idades entre 10 e 12 anos, usuárias desses parques. Todos os participantes foram informados do objetivo e da metodologia pretendida no estudo, da participação espontânea e voluntária, da garantia de sigilo e de anonimato. Foi obtido o consentimento informado de todos os participantes e de seus responsáveis de educação/pais. Participaram dos GFs as crianças que retornaram ambos os documentos devidamente preenchidos e assinados. Os GFs se deram numa sala da escola pública vizinha do parque, tiveram duração média de 35 minutos e foram gravados em registro áudio, tendo sido feitas anotaçôes pela entrevistadora durante a realização. Os encontros foram únicos, com um mesmo guião semiestruturado para todos os GFs e moderados sempre pela mesma entrevistadora, que não possuía qualquer vínculo com os participantes. $\mathrm{O}$ incentivo à participação de todos foi constante, sendo registrada a atuação ativa das crianças ao longo de todo o encontro.

Para a análise desses dados, procedeu-se a uma transcrição integral das perguntas e respostas de cada GF, assinalando pausas e interjeiçóes, e optou-se pelo tema como 
unidade na análise de conteúdo. Foi realizada uma análise indutiva com o recurso ao programa QSR NVivo 11 para a sua gestão e codificação, de forma independente, por duas das autoras, tendo uma a responsabilidade da edição do processo de codificação. Os resultados foram comparados e discutidos até ser obtido um consenso. Os procedimentos de análise e discussão foram acompanhados por outra terceira autora no sentido de promover o confronto de crenças, valores e preconceitos.

\section{Resultados}

\section{Caracterização das estruturas dos parques}

As características das estruturas dos parques da amostra são apresentadas na tabela 1 , na qual se verifica a oferta de diferentes estruturas entre parques.

Tabela 1. Caracterização estrutural dos parques

\begin{tabular}{|c|c|c|}
\hline & P1 & $\mathrm{P} 2$ \\
\hline Tamanho & 30 ha & $2.5 \mathrm{ha}$ \\
\hline \multirow[t]{12}{*}{ Estruturas } & 8 "Open spaces" & \\
\hline & Trilha & Trilha \\
\hline & (não pavimentada) & (não pavimentada) \\
\hline & 2 parques infantis & 1 parque infantil \\
\hline & 1 campo de futebol & 2 quadras de tênis \\
\hline & 1 estação “fitness" & 2 quadras polidesportivas (desativadas) \\
\hline & 1 área "street workout" & 11 quadras de petanca ("pieds tanqués") \\
\hline & 1 percurso atlético & 1 área de "pic nic" \\
\hline & 1 área de "pic nic" & 1 área de praia fluvial \\
\hline & 1 lago & Estacionamento (carro) \\
\hline & Banheiro & \\
\hline & $\begin{array}{l}\text { Estacionamento (carros e } \\
\text { bicicletas) }\end{array}$ & \\
\hline
\end{tabular}


$\mathrm{O}$ P1, inserido numa região central de Guimarães, destaca-se pela maior dimensão (30 ha; tabela 1) e ampla área verde numa zona residencial urbana. Localizado ao lado de uma escola pública, possui dois parques infantis, que podem favorecer a maior frequência de utilização pelas crianças. Essas duas áreas do P1 estão localizadas em áreas diferentes e são compostas por equipamentos distintos, sendo que um dos parques infantis possui estruturas tradicionais, com balanços, escorregadores e plataformas (figura 1); e o outro, estruturas diferentes das tradicionais, que podem desafiar e atrair o público infantil, como cordas, "teias", percursos, argolas para pendurar-se. A disponibilidade de informaçóes sobre o parque (pontuação EAPRS = 3), a presença de percursos através do parque (pontuação EAPRS $=32$ ) e a melhor acessibilidade (pontuação EAPRS $=98$ ) foram características avaliadas que se destacaram no P1.

Figura 1. Imagem do parque infantil do P1

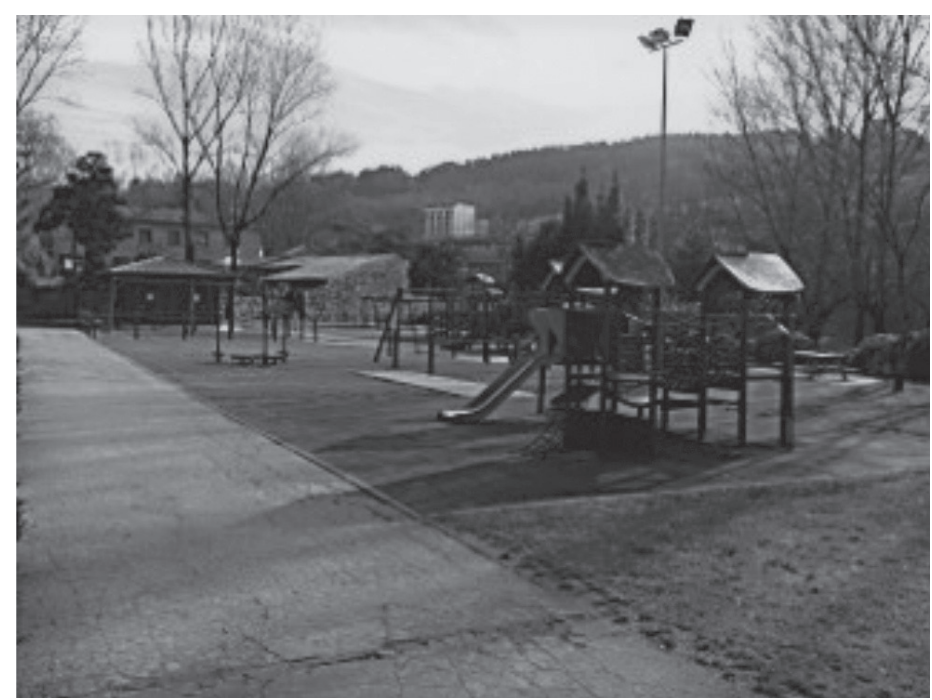

Foto: Simone Medeiros de Oliveira.

O P2 está localizado nas margens de um rio (rio Ave), tendo sido no passado uma área balnear, condição que pode torná-lo atraente, favorecendo a maior frequência de utilizaçáo do parque pelos indivíduos (figura 2). É um parque de menor dimensão (2.5 ha; tabela 1), mas que se destacou pela oferta de 11 quadras de petanca (pontuação EAPRS = 120; figura 3), modalidade desportiva semelhante 
à bocha. Conforme se verifica na figura 3, a ausência de facilidades de apoio aos usuários (banheiro, ponto de encontro, regras de utilização, mapas, bicicletário e mesas) foi uma característica avaliada (pontuação EAPRS $=0$ ) que pode limitar a frequência de utilização desse parque pelas crianças.

Figura 2. Imagem da área balnear do P2

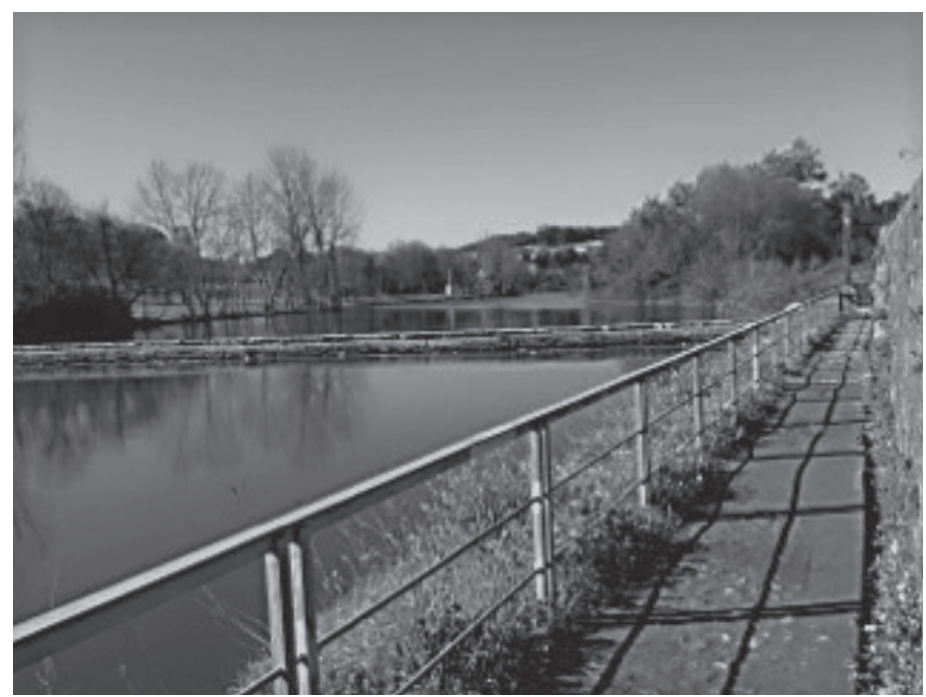

Foto: Simone Medeiros de Oliveira.

A qualidade das estruturas dos parques (figura 3) apresentou uma semelhança entre a amostra em relação à estética geral (pontuação EAPRS P1 = 47; pontuação EAPRS P2 $=46$ ) e à paisagem (pontuação EAPRS P1 = 29; pontuação EAPRS $\mathrm{P} 2$ = 24). Todavia, em ambos se registrou a ausência de trilha pavimentada e de facilidades relacionadas com a segurança dos parques (telefones públicos). 
Figura 3. Qualidade das estruturas dos parques

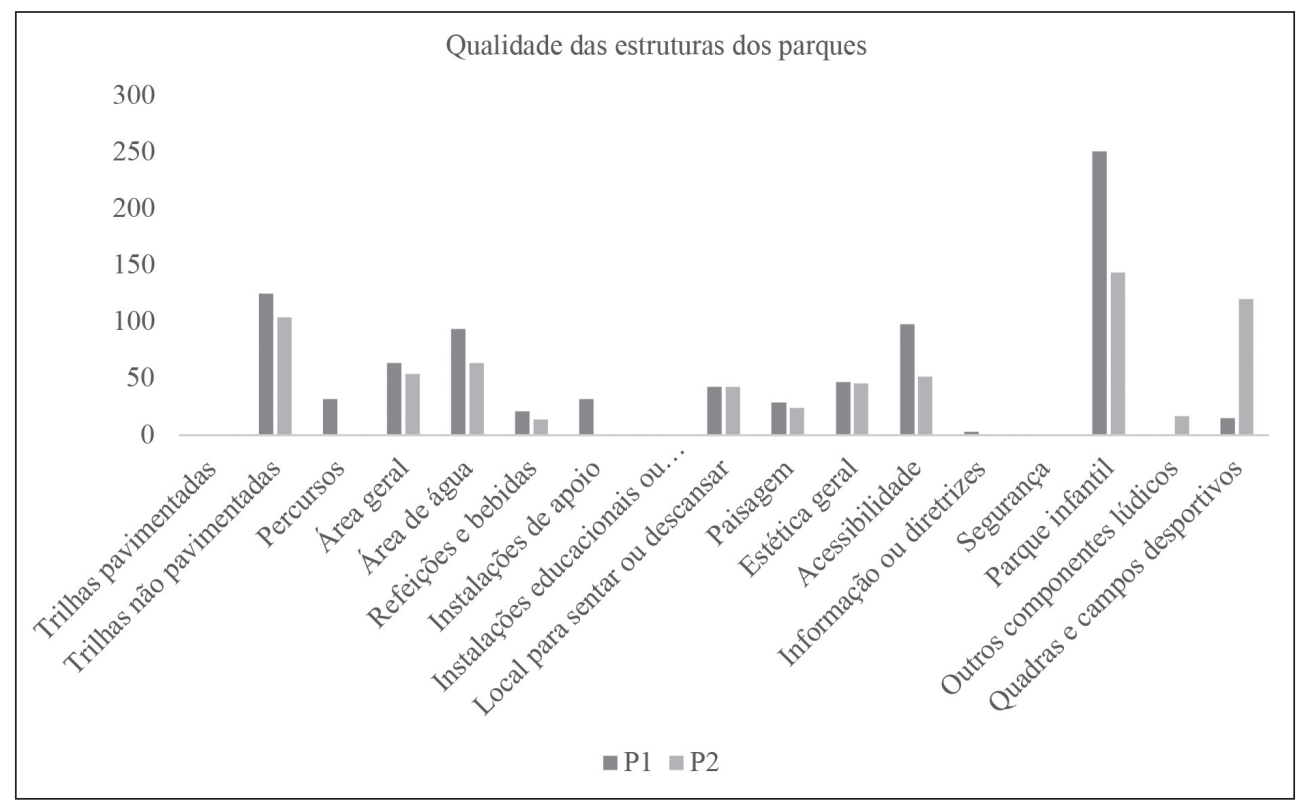

Fonte: elaborado pelas autoras.

\section{Dados qualitativos}

Através da análise dos dados dos GFs, foram identificados quatro temas e correspondentes subtemas relacionados à frequência de utilização dos parques pelas crianças (tabela 2): utilização dos parques, segurança dos parques, oferta das estruturas e necessidades percebidas pelas crianças. 
Tabela 2. Temas e subtemas emergentes da análise do conteúdo das entrevistas

\begin{tabular}{cc}
\hline Tema & Subtemas \\
\hline Utilização & Companhia \\
& Passear o cão \\
Socializaçấo
\end{tabular}

Fonte: Quatro grupos focais com crianças (10-12 anos).

\section{Utilização dos parques}

A companhia das crianças nos parques esteve centrada na figura de um adulto, em geral familiar, evidenciando o suporte parental como um fator associado à frequência de utilização dos parques pelas crianças: "Eu jogo com o meu pai futebol, brincamos, dizemos algumas piadas para nos entretermos, às vezes" (menina). "Eu vou de bicicleta, a minha mãe também corre e eu vou de bicicleta com o meu pai” (menino).

A oportunidade de AF nos parques surgiu nos relatos das crianças, inclusive quando supervisionadas por adultos que adotam comportamentos sedentários: "Enquanto o meu pai está a trabalhar no telemóvel e eu estou a passear o meu cão" (menino). "Às vezes, meus pais ficam lá sentados numa rocha e eu prefiro brincar, mas às vezes meu pai também vai lá comigo para me ajudar a fazer certas coisas" (menina).

Passear o cáo surgiu como um potencial fator para a maior frequência de utilizaçáo dos parques pelas crianças: "Às vezes, nas férias um casal de amigos deixa-nos lá uma cadela e nós íamos ao parque assim com o cão" (menina). "À beira da minha casa há um parque e eu vou com o meu cão quando vou sozinho” (menino). 
A socialização nos parques, considerada um benefício da utilização desses ambientes naturais, foi percebida pelas crianças dos GFs: "Mas também é mais divertido ir com a família, para convivermos mais e assim" (menina).

Assim, verifica-se que a utilização dos parques pelas crianças entrevistadas é realizada de forma familiar, sendo que as atividades são distintas entre crianças e adultos. O suporte de um adulto e a posse de um animal de estimação podem favorecer a frequência de utilização dos parques pelas crianças.

\section{Segurança dos parques}

Nos relatos das crianças, a percepção de segurança no parque destacou-se como principal fator associado à frequência de utilização dos parques pelas crianças. A maioria percebe o parque como local não seguro, frequentando-o preferencialmente na companhia de outrem: "Eu vou sozinha se for preciso, mas prefiro ir com a família" (menina). "Eu acho que não tem segurança se uma pessoa for sozinha" (menino).

A presença de um vigilante foi referida pelas crianças como uma estratégia que aumenta a segurança nos parques, além de contribuir para a sua manutenção: "Eu acho que devia haver seguranças para não, não nos sentirmos (silêncio)” (menino). "Mas também é por isso que também tem que haver guardas lá. Bem por isso, para não haver vandalismo" (menino).

A segurança percebida pelas crianças sobre as estruturas ofertadas nos parques foi outro fator que surgiu nos relatos e que pode afetar a frequência e inibir a AF das crianças nos parques: "Não, eu acho que podiam renovar por exemplo o escorrega, o baloiço, porque a poucos dias ali estava a andar um menino, a corda arrebentou, ele caiu e aleijou-se" (menino). "Por exemplo este parque tem um rio, uma pessoa vai buscar a bola e desequilibra-se, pode cair" (menino).

Segundo as crianças, a quantidade de usuários dos parques também seria um aspecto relacionado com a segurança dos parques: "Segurança até atrai mais pessoas" (menina).

Dessa forma, estes resultados evidenciam o impacto da percepção sobre a segurança nos parques, que pode condicionar a sua utilização pelas crianças. Parques percebidos como seguros podem favorecer a frequência de utilização por elas, aumentando a oportunidade de elas serem mais ativas. 


\section{Oferta das estruturas}

As crianças relataram satisfaçáo na oferta das estruturas existentes nos parques da amostra: "Eu acho se for referido para desporto, eu acho que é suficiente porque tem pistas próprias, tem relva, depois tem aquelas pistas, depois tem parques para as crianças mais pequenas [...]" (menina). "E tem espaço suficiente para as pessoas fazerem o que quiserem" (menino).

Entretanto, a manutenção e a renovação das estruturas surgiram como uma questão que pode afetar a frequência de utilização dos parques pelas crianças. A falta ou a má manutenção das estruturas pode impedir a sua utilização por elas, inibindo a adoção de comportamentos fisicamente ativos: "Eu acho que quando chove e depois quando há sol deviam limpar o escorrega e assim, porque depois nós queremos ir para lá brincar e andar de baloiço e não dá” (menina).

A limpeza dos parques foi um item ressaltado pelas crianças, em geral, relacionado aos comportamentos dos usuários dos parques: “[... também acho que, por exemplo, os parques poderiam estar mais limpos" (menina). "[...] por muito que limpem, as pessoas estấo sempre a deitar lixo para o chão. Tanto que (pausa), mas que continuam a limpar cada vez mais porque as pessoas não têm o costume, muitas delas não têm noção do que estão a fazer" (menina). "Cáes. Eles fazem o cocô e depois não apanham. Depois uma pessoa vai lá andar e calca [...]” (menino). Estas preocupaçóes das crianças sugerem uma consciência e atitude pró-ambientais derivadas da frequência de utilização desses ambientes naturais. A sua crítica recai sobre as pessoas que utilizam os parques bem como sobre a insuficiência de estruturas de apoio como depósito para o lixo: "Eu também acho que podia haver mais caixote de lixo que chamassem mais atenção para que as pessoas não deitassem, não deitassem sempre ao chão" (menino). "Se tivesse mais coisa de reciclagem era muito melhor" (menino).

Curiosamente, as crianças entrevistadas percebem que um maior controle no cumprimento das regras nos parques poderia minimizar algumas fragilidades dos parques percebidas pelas mesmas: "Eu acho que também deviam haver regras para entrar aos parques" (menina). "Tem que haver regras no parque, tipo, não deitar lixo para o chão" (menino).

Os relatos revelam a satisfação das crianças com a oferta disponível nos parques da amostra, sendo reconhecida como suficiente. Todavia, a manutenção destacou-se nos depoimentos, revelando o potencial deste fator sobre a frequência das crianças nos parques. 


\section{Necessidades percebidas pelas crianças}

Algumas das necessidades percebidas pelas crianças em relação aos parques envolvem itens relacionados ao tema anteriormente apresentado. Importante ressaltar a percepção das crianças em relação à necessidade da presença de um vigilante, o que, segundo as crianças, asseguraria, além da segurança, a manutenção e o apoio aos usuários dos parques:"Devia ter vigilantes por causa dos grandes que ocupam as máquinas. E depois, as crianças querem brincar e chegam lá e aquilo lá está partido e [...]" (menino). "Eu acerca disso acho também que devia, porque quando as pessoas se magoam não tem pessoas por perto para (pausa), devia ter um guarda lá e caixas telefónicas lá, para ligar para o 112" (menino).

A diversidade de estruturas e atividades organizadas foram fatores percebidos pelas crianças que podem atrair mais crianças aos parques pela oportunidade de realizar experiências diversificadas: "Devia de haver mais (pausa), no parque devia de haver, ter lá muitas atraçóes como o parque de cordas, vários baloiços, muito escorregas" (menina). "Eu acho que havia de haver lá sempre uma pessoa a fazer atividades por exemplo do zumba, tipo às vezes trazia arcos, outras vezes boxe" (menina).

A oferta de entretenimento para os adultos acompanhantes das crianças nos parques pode estar associada à frequência das crianças a esses ambientes. Através dos relatos das crianças, identificou-se a preocupação delas em proporcionar bons momentos aos adultos enquanto estes os acompanham nos parques: "[...] talvez um espaço público em que pudessem estar os pais [...]. Para que os pais pudessem estar a vigiar as crianças e ao mesmo tempo estar com, com outras pessoas da idade deles ou a divertir-se também. Porque eu acho que os adultos também têm direito [...]" (menina). "Também havia de haver um spa para (pausa), para as mulheres adultas porque, enquanto que os filhos estão a brincar, podem estar a se embelezar" (menino).

A disponibilidade de material recreativo nos parques náo foi valorizada pelas crianças. Mas a possibilidade desta esteve associada ao empréstimo na forma de aluguel: "Se tivesse uma bola e tivéssemos, olha é um euro toma e nós metíamos lá e quando acabássemos metíamos lá outra vez" (menino). "Alugavas uma bola. E tipo ias brincar" (menina).

Curiosamente, uma necessidade percebida pelas crianças foi o tipo de solo disponível nos parques. Segundo os relatos, a oferta de solos mais adequados pode contribuir para uma melhor utilizaçáo dos parques pelas crianças, favorecendo o 
aumento da frequência delas nesses ambientes: "Pôr sintética, porque depois para ir lá é meio (pausa), quando chove aquilo é horrível" (menino). "Se chover, aquilo fica encharcado e se vai para lá e depois só meter lá o coisa (refere-se ao pé) e já escorrega. Pois, devia ter relva pelo menos" (menino).

A oportunidade de utilização dos parques para práticas desenvolvidas pelas escolas surgiu nos relatos das crianças como uma possibilidade de maior frequência nos parques: "Acho que (pausa), acho que o parque como ela disse, devia haver mais modalidades para a escola também incentivar as crianças a irem aos parques, a levarem nós mesmo. Aqui às vezes, mesmo em vez de termos às vezes, por mês, imagine uma aula $\mathrm{A}, \mathrm{B}$ ou $\mathrm{C}$, vamos ao parque fazer qualquer coisa ou então uma aula de Física (refere-se à Educação Física) e vamos para lá” (menino).

Foi citada a condição favorável da proximidade entre o parque e a escola como uma oportunidade de maior frequência de utilização dos parques, evitando inclusive a adoção de alguns comportamentos sedentários, atualmente comuns às crianças, reconhecidos pelas mesmas: "Eu acho que devia haver parques à beira das escolas, porque assim, porque as crianças, os pais vêm buscar tarde e então enquanto, ao invés de nós estarmos aqui na escola e irmos para os computadores e isso, poderíamos sair da escola, estava lá um vigilante e irmos para o parque e começarmos a fazer exercício físico, a brincar, invés do que estar metidos na escola e nos computadores, enquanto que esperamos pelos pais. É isso" (menina).

Interessantemente, a divulgação dos parques foi percebida pelas crianças como uma necessidade para o aumento da frequência de utilização dos parques: "Devia de haver alguma coisa que chamasse a atenção de todos no parque, para virem todos, para não estarem, aquilo está tudo deserto" (menino).

A presença de um vigilante e a oferta diversificada de estruturas e atividades são condiçôes que poderão aumentar a percepção das crianças sobre os parques como um ambiente seguro e atraente, favorecendo a maior frequência de utilização pelas crianças e pelas escolas.

\section{Discussão}

No presente estudo, a percepção da necessidade de maior segurança nos parques para um aumento da frequência de utilização pelas crianças ficou demonstrada no discurso dessas. A percepção de segurança é um fator social evidenciado como um 
mediador da utilização dos ambientes naturais, em especial dos parques (KRUGER et al., 2010; OU et al., 2016). Estratégias que assegurem melhores condições aos parques poderão contribuir para a melhor percepção de segurança e maior frequência de utilização desses ambientes pelas crianças.

A presença de vigilantes nos parques, sugerida pelas crianças, é uma estratégia que poderá melhorar a percepção de segurança nos parques e, consequentemente, aumentar a frequência de utilização dos parques pelas crianças para a prática da AF. Um estudo realizado com adolescentes norte-americanos verificou a associação entre a percepção de segurança no parque e a prática de AF nesses ambientes. Com efeito, os adolescentes que reportaram maior segurança foram os mais engajados em práticas de AF nesses ambientes (BABEY et al., 2015), sugerindo uma relação entre a AF praticada nos parques e a segurança percebida pelos usuários nesses locais. Todavia, em outro estudo também realizado em parques nos Estados Unidos, a presença de um vigilante e a percepção de segurança dos usuários não foram associadas à frequência de utilização dos parques (COHEN et al., 2010). Esta necessidade de reforço da segurança nos parques, segundo as crianças entrevistadas, revela fragilidades locais que podem afetar a frequência de utilização dos parques pelas crianças para a prática da AF. A exemplo do registro da ausência de estruturas relacionadas com a segurança em ambos os parques (pontuação EAPRS $=0$ ) $\mathrm{e}$ dos relatos de utilizaçáo dos parques na companhia de adultos. A elaboração de estratégias que proporcionem maior suporte ao nível de estruturas e ao nível social local poderá favorecer o aumento da frequência de utilização dos parques pelas crianças para a prática da AF.

A análise dos relatos das crianças entrevistadas sobre a necessidade de medidas de segurança nos parques ratifica a percepçáo de insegurança dessas nos parques e sugere a extensão desta percepção aos adultos responsáveis pelas mesmas. A relação entre a percepção parental sobre o ambiente e o comportamento fisicamente ativo das crianças é conhecida, sendo que ambientes percebidos como pouco seguros pelos pais estão associados a menor mobilidade independente das crianças (SANTOS et al., 2013). A frequência de utilização dos parques pelas crianças de forma independente poderia contribuir para o aumento da utilização dos parques pelas crianças e para a AF diária das mesmas. Porém, os resultados deste estudo sugerem que os parques da amostra são percebidos como ambientes pouco seguros, visto que as crianças relatam a utilização a esses ambientes na companhia de um adulto, condição que 
parece refletir uma menor mobilidade independente das crianças aos parques. Esta necessidade de maior segurança nos parques poderá decorrer de uma educação num contexto marcadamente adultocêntrico, que requer a presença/vigilância de um adulto em todas as atividades das crianças. Pelos relatos das crianças, esta necessidade não surge de forma pessoal, mas como algo imposto pelo contexto, sendo de realçar a falta de justificaçáo das crianças para esta percepção de falta de segurança nos parques.

As crianças relataram predominantemente a adoção de comportamentos fisicamente ativos nos parques. A supervisão de um adulto está associada a uma menor probabilidade desses comportamentos (FLOYD et al., 2011). Entretanto, o estilo de conduta parental é um fator associado aos níveis de intensidade da AF diária das crianças, sendo que filhos de mães permissivas podem apresentar um maior nível de AF diária (JAGO et al., 2011). De maneira que a companhia de um adulto com condutas menos controladoras poderá contribuir para a maior prática da AF das crianças nos parques. Segundo um estudo recente (HNATIUK et al., 2016), entre os níveis ambientais nos quais as crianças estão inseridas, o social é o que tem maior impacto sobre a AF das crianças no meio doméstico e nos arredores da residência, podendo estar relacionado com o padrão de conduta parental, moldado a partir do ambiente social no qual a criança está inserida. Assim, a reflexão sobre o ambiente social e suas possíveis influências sobre o estilo de conduta parental deve ser incluída na análise da AF praticada pelas crianças nos parques. A influência parental ou familiar sobre a AF das crianças é um fator já muito comprovado no meio científico. $\mathrm{O}$ suporte parental é um fator associado à $\mathrm{AF}$, reconhecido pelas crianças (ROTH et al., 2012), que colabora positivamente para a AF diária das crianças e negativamente para a adoção de comportamentos sedentários nas crianças (MCMINN et al., 2013; TANDON et al., 2014; LAU et al., 2015).

A presença de um parque vizinho à escola pode ser otimizada com a maior utilização pelas escolas. Evidências (GRAZIOSE et al., 2016) revelam que o acesso ao parque vizinho à escola pode contribuir para a prática da AF e a saúde mental das crianças. De maneira que a maior utilização do parque vizinho pelas escolas poderá ser uma estratégia que beneficie a saúde das crianças, através da oportunidade de AF e contato com a natureza. Neste estudo, as crianças criticam a escola por não lhes proporcionar a utilização dos parques vizinhos, o que diminuiria a adoção de comportamentos sedentários no período escolar, além de tornar a sua rotina diária 
mais atraente e de contribuir para descentralização da utilização dos parques pelas crianças na companhia dos pais ou familiares.

A manutenção dos parques também surgiu nos resultados do estudo como um fator que poderá interferir na frequência das crianças nos parques para a prática da AF. A má conservação dos equipamentos nos parques foi percebida pelas crianças como uma barreira para a utilização dos mesmos, refletindo em menores oportunidades de AF. Flowers et al. (2016) destacam a relação entre a percepção da qualidade da oferta e a frequência dos indivíduos nos parques. Já Cohen et al. (2016) verificaram que as condiçôes dos parques podem potencializar a AF praticada pelos indivíduos. Entretanto, a renovação dos parques, além de colaborar para a maior frequência de crianças, está associada a um aumento da percepção de segurança dos seus usuários (COHEN et al., 2015). A manutenção adequada dos parques surge como elemento facilitador da frequência e da AF das crianças nesses ambientes, favorecendo simultaneamente a percepção de maior segurança de seus usuários.

Curiosamente, a posse de um cão como animal de estimação surgiu nos relatos das crianças como uma oportunidade de maior frequência de utilização dos parques. Segundo Evenson et al. (2016), passear o cão nos parques pode cooperar para a AF dos adultos, todavia, é um comportamento que tem pouca influência sobre os níveis de AF praticada no parque. Dessa forma, os dados do presente estudo sugerem que ter um cão como animal de estimação pode contribuir para a maior frequência de utilização dos parques pelas crianças. Por sua vez, a companhia de pares no parque não teve destaque entre os relatos das crianças dos GFs. Estudos revelam que as crianças se agrupam de acordo com os níveis de AF que praticam (MACDONALDWALLIS et al., 2011) e que nos parques, a presença de outra criança ativa pode aumentar os níveis de AF praticada pela criança (FLOYD et al., 2011). Assim, as estratégias que aumentem a frequência infantil de utilização dos parques são válidas, sendo que os parques percebidos como seguros podem aumentar a oportunidade de convívio entre pares de forma fisicamente mais ativa.

Este estudo apresenta pontos fortes e fracos. A caracterização e a avaliação das estruturas dos parques possibilitaram uma aproximação da realidade percebida pelas crianças que fundamentou as conclusões do estudo. Uma ampliação da amostra de parques e respectivos GFs poderia contribuir com novas realidades e, além disso, a participação de adolescentes neste estudo também complementaria os resultados com perspectivas diferentes. 
Os resultados deste estudo não podem ser generalizados para todas as populaçôes, tendo em vista as diferentes realidades decorrentes das políticas empregadas, do ambiente e da cultura local, que têm impacto sobre as perceçôes e os comportamentos dos indivíduos. Todavia, estes resultados são relevantes para a construção de políticas de promoção da AF das crianças em parques.

\section{Considerações finais}

Este estudo contribui para a reflexão sobre os ambientes naturais e as oportunidades de comportamentos fisicamente ativos em crianças. Proporcionar a utilização desses ambientes favorecendo a prática de AF das crianças pode colaborar para a prevenção de doenças decorrentes do estilo de vida sedentário e para o cumprimento das recomendações de práticas diárias de AF. Os parques são ambientes naturais que podem potencializar as oportunidades de AF das crianças por proporcionarem condiçôes que facilitam a adoção deste comportamento.

A segurança percebida nos parques surgiu como uma forte evidência para a frequência da sua utilização pelas crianças. Condiçôes que as crianças percepcionem como aumento da segurança nos parques poderão aumentar a sua frequência nos parques. A companhia de um cão como animal de estimação também é outro fator que pode cooperar para o aumento da frequência nesses locais. A manutenção dos equipamentos deve reger uma das açóes primárias dos setores envolvidos com a urbanização das cidades. Políticas de incentivo para maior utilização dos parques pelas escolas e a oferta de parques localizados nas áreas residenciais das crianças podem contribuir para a descentralização da figura parental e/ou familiar como companhia para a frequência das crianças nos parques, bem como para a prática de AF entre pares.

Incentivos públicos à frequência de utilização dos parques de forma ativa devem centrar suas atençôes sobre os fatores de segurança e manutenção das estruturas dos parques, em especial quando a população-alvo for crianças. Criar mais oportunidades de frequência das crianças de forma independente nos parques pode potencializar os contributos desses ambientes na adoção de comportamentos ativos. ${ }^{1}$ 


\section{Referências}

ADINOLFI, C.; SUÁREZ-CÁCERES, G. P.; CARIÑANOS, P. Relation between visitors' behaviour and characteristics of green spaces in the city of Granada, south-eastern Spain. Urban Forestry \& Urban Greening v. 13, p. 534-542, Jan. 2014. Disponível em: <http://search. ebscohost.com/login.aspx?direct=true $\& \mathrm{db}=$ edselp\&AN=S1618866714000351\&lang=ptbr\&site $=$ eds-live $\&$ scope $=$ site $>$.

BABEY, S. H. et al. Neighborhood, family and individual characteristics related to adolescent park-based physical activity. Preventive Medicine, v. 76, p. 31-36, July 2015. Disponível em: <http://search.ebscohost.com/login.aspx?direct=true $\& d b=$ edselp \&AN=S0091743515001012\& lang=pt-br\&site $=$ eds-live\&scope $=$ site $>$.

BARAN, P. K. et al. Park Use Among Youth and Adults: Examination of Individual, Social, and Urban Form Factors. Environment and Behavior, v. 46, n. 6, p. 768-800, 2014. Disponível em: <http://www.scopus.com/inward/record.url?eid=2-s2.0-84903973570\&partnerID=40\&m d5=b25207e3b442275d44ebe793dce484c0>.

BEDIMO-RUNG, A. L.; MOWEN, A. J.; COHEN, D. A. The significance of parks to physical activity and public health: a conceptual model. Am J Prev Med, v. 28, n. 2, supl 2, p. 159-68, Feb. 2005. Disponível em: <http://ac.els-cdn.com/S0749379704003046/1-s2.0S0749379704003046-main.pdf?_tid=d940a7f2-c214-11e6-84f1-00000aacb360\&acdnat=148 1730728_7bdefble9f778dd34bb82df5343a95ca>.

BOHN-GOLDBAUM, E. E. et al. Does playground improvement increase physical activity among children? A quasi-experimental study of a natural experiment. J Environ Public Health, v. 2013, p. 109841, 2013. Disponível em: <https:/www.ncbi.nlm.nih.gov/pmc/articles/ PMC3694497/pdf/JEPH2013-109841.pdf>.

CASPERSEN, C. J.; POWELL, K. E.; CHRISTENSON, G. M. Physical activity, exercise, and physical fitness: definitions and distinctions for health-related research. Public Health Reports, v. 100, n. 2, p. 126-131, Mar.-Apr. 1985. Disponível em: <http://www.ncbi.nlm.nih. gov/pmc/articles/PMC1424733/>.

COHEN, D. A. et al. Impact of park renovations on park use and park-based physical activity. J Phys Act Health, v. 12, n. 2, p. 289-95, Feb. 2015. Disponível em: <http://www.ncbi.nlm.nih. gov/pmc/articles/PMC4851467/pdf/nihms737230.pdf>.

. Parks and physical activity: Why are some parks used more than others? Preventive Medicine, v. 50, Supplement, p. S9-S12, 2010. Disponível em: <http://www.sciencedirect.com/ science/article/pii/S009174350900485X>. 
. Research Article: The First National Study of Neighborhood Parks. Implications for Physical Activity. American Journal of Preventive Medicine, Jan. 2016. Disponível em: <http:// search.ebscohost.com/login.aspx?direct=true \&db=edselp\&AN=S0749379716300794\&lang= pt-br\&site=eds-live \&scope $=$ site $>$.

DUNTON, G. F. et al. Neighborhood park use by children: use of accelerometry and global positioning systems. Am J Prev Med, v. 46, n. 2, p. 136-42, Feb. 2014. Disponível em: <http:// ac.els-cdn.com/S0749379713005825/1-s2.0-S0749379713005825-main.pdf?_tid=c897861a3c7b-11e6-a664-00000aacb360\&acdnat=1467041482_68f57e4fc6d214081caaf1f428ca6ef8>.

EVENSON, K. R. et al. Use of Dog Parks and the Contribution to Physical Activity for Their Owners. Res Q Exerc Sport, v. 87, n. 2, p. 165-73, Jun. 2016. Disponível em: <http://www. tandfonline.com/doi/pdf/10.1080/02701367.2016.1143909>.

FLOWERS, E. P.; FREEMAN, P.; GLADWELL, V. F. A cross-sectional study examining predictors of visit frequency to local green space and the impact this has on physical activity levels. BMC Public Health, v. 16, n. 1, p. 420, 2016. Disponível em: <http://www.ncbi.nlm.nih. gov/pmc/articles/PMC4875629/pdf/12889_2016_Article_3050.pdf>.

FLOYD, M. F. et al. Park-based physical activity among children and adolescents. Am J Prev Med, v. 41, n. 3, p. 258-65, Sep. 2011. Disponível em: <http://ac.els-cdn.com/S0749379711003278/1s2.0-S0749379711003278-main.pdf?_tid=d9bd315e-4781-11e6-93d2-00000aab0f268acdnat $=1468253551 \_357 f 20$ a357043ba67894357089033c02>.

GLADWELL, V. F. et al. The great outdoors: how a green exercise environment can benefit all. Extrem Physiol Med, v. 2, n. 1, p. 3, 2013. Disponível em: <http://www.ncbi.nlm.nih.gov/pmc/ articles/PMC3710158/pdf/2046-7648-2-3.pdf>.

GRAZIOSE, M. M. et al. Association Between the Built Environment in School Neighborhoods With Physical Activity Among New York City Children, 2012. Prev Chronic Dis, v. 13, p. E110, Aug. 18 2016. Disponível em: <https:/www.ncbi.nlm.nih.gov/pmc/articles/PMC4993120/ pdf/PCD-13-E110.pdf>.

HAN, B.; COHEN, D.; MCKENZIE, T. L. Quantifying the contribution of neighborhood parks to physical activity. Preventive Medicine, v. 57, p. 483-487, 11/1/November 20132013. Disponível em: <http://search.ebscohost.com/login.aspx?direct=true\&db=edselp\&AN=S00917 43513002132\&lang=pt-br\&site $=$ eds-live $\&$ scope $=$ site

HARTIG, T. et al. Nature and Health. In: FIELDING, J. E. (Ed.). Annual Review of Public Health, v. 35, p. 207, 2014.

HNATIUK, J. A.; HESKETH, K. R.; VAN SLUijS, E. M. Correlates of home and neighbourhood-based physical activity in UK 3-4-year-old children. Eur J Public Health, May 11 2016. Disponível em: <http://eurpub.oxfordjournals.org/content/eurpub/early/2016/05/10/ eurpub.ckw067.full.pdf>. 
JAGO, R. et al. Parenting styles, parenting practices, and physical activity in 10- to 11year olds. Prev Med, v. 52, n. 1, p. 44-7, Jan. 2011. Disponível em: <http://ac.els-cdn.com/ S0091743510004354/1-s2.0-S0091743510004354-main.pdf?_tid=75dded08-c37a-11e5-b67600000aab0f6b\&acdnat=1453736823_6573eabdcabe4057a3b1c54be861e4c6>.

KACZYNSKI, A. T.; HENDERSON, K. A. Parks and recreation settings and active living: a review of associations with physical activity function and intensity. J Phys Act Health, v. 5, n. 4, p. 619-32, Jul. 2008.

KLINKER, C. D. et al. Context-Specific Outdoor Time and Physical Activity among SchoolChildren Across Gender and Age: Using Accelerometers and GPS to Advance Methods. Front Public Health, v. 2, p. 20, 2014a. ISSN 2296-2565. Disponível em: <http://www.ncbi.nlm.nih. gov/pmc/articles/PMC3949325/pdf/fpubh-02-00020.pdf>.

. Using accelerometers and global positioning system devices to assess gender and age differences in children's school, transport, leisure and home based physical activity. Int J Behav Nutr Phys Act, v. 11, p. 8, 2014b. ISSN 1479-5868. Disponível em: <http://www.ncbi.nlm.nih. gov/pmc/articles/PMC3905652/pdf/1479-5868-11-8.pdf>.

KRUGER, J.; LANKFORD, T.; SCHMID, T. State legislative support for parks. Environ Health Insights, v. 4, p. 27-31, 2010. Disponível em: <http://www.la-press.com/redirect_file. php?fileId=2877\&filename=2092-EHI-State-Legislative-Support-for-Parks.pdf\&fileType=pdf $>$.

KURKA, J. M. et al. Patterns of neighborhood environment attributes in relation to children's physical activity. Health Place, v. 34, p. 164-70, Jul. 2015. Disponível em: <http://www. sciencedirect.com/science/article/pii/S1353829215000726>.

LARSON, L. R.; JENNINGS, V.; CLOUTIER, S. A. Public Parks and Wellbeing in Urban Areas of the United States. PLoS One, v. 11, n. 4, p. e0153211, 2016. Disponível em: <https:// www.ncbi.nlm.nih.gov/pmc/articles/PMC4824524/pdf/pone.0153211.pdf>.

LAU, E. Y. et al. Associations Between Home Environment and After-School Physical Activity and Sedentary Time Among 6th Grade Children. Pediatr Exerc Sci, v. 27, n. 2, p. 226-33, May 2015. Disponível em: <https:/www.ncbi.nlm.nih.gov/pmc/articles/PMC4426238/pdf/ nihms673861.pdf>.

LEE, A. C.; JORDAN, H. C.; HORSLEY, J. Value of urban green spaces in promoting healthy living and wellbeing: prospects for planning. Risk Manag Healthc Policy, v. 8, p. 131-7, 2015. Disponível em: <https://www.dovepress.com/getfile.php?fileID=26774>.

MACDONALD-WALLIS, K. et al. School-based friendship networks and children's physical activity: A spatial analytical approach. Soc Sci Med, v. 73, n. 1, p. 6-12, Jul. 2011. Disponível em: <http://ac.els-cdn.com/S0277953611002553/1-s2.0-S0277953611002553-main.pdf?_ tid=785ccae0 - c37a-11e5-8a75-00000aab0f6c\&acdnat=1453736827_f87c76df9dc1879d53904 f1977825ec5>. 
MCCRACKEN, D. S.; ALLEN, D. A.; GOW, A. J. Associations between urban greenspace and health-related quality of life in children. Prev Med Rep, v. 3, p. 211-21, Jun. 2016. Disponível em: <https://www.ncbi.nlm.nih.gov/pmc/articles/PMC4929180/pdf/main.pdf>.

MCMINN, A. M. et al. Family and home influences on children's after-school and weekend physical activity. Eur J Public Health, v. 23, n. 5, p. 805-10, Oct. 2013. Disponível em: <https:// www.ncbi.nlm.nih.gov/pmc/articles/PMC3784797/pdf/cks160.pdf>.

MITCHELL, C. A.; CLARK, A. F.; GILLILAND, J. A. Built Environment Influences of Children's Physical Activity: Examining Differences by Neighbourhood Size and Sex. Int J Environ Res Public Health, v. 13, n. 1, 2016. Disponível em: <http://www.mdpi.com/16604601/13/1/130/pdf>.

OU, J. Y. et al. A Walk in the Park: The Influence of Urban Parks and Community Violence on Physical Activity in Chelsea, MA. Int J Environ Res Public Health, v. 13, n. 1, Jan. 42016. Disponível em: <http://www.mdpi.com/1660-4601/13/1/97/pdf>.

PEARCE, M. et al. Who children spend time with after school: associations with objectively recorded indoor and outdoor physical activity. International Journal of Behavioral Nutrition and Physical Activity, v. 11, n. 1,p. 45, 2014.

PERRY, C. K. et al. Places where children are active: A longitudinal examination of children's physical activity. Prev Med, v. 93, p. 88-95, Dec. 2016. Disponível em: <http://www. sciencedirect.com/science/article/pii/S0091743516302730>.

RICHARDSON, E. A. et al. The role of public and private natural space in children's social, emotional and behavioural development in Scotland: A longitudinal study. Environ Res, v. 158, p. 729-736, Oct. 2017.

ROTH, M. A.; MILLETT, C. J.; MINDELL, J. S. The contribution of active travel (walking and cycling) in children to overall physical activity levels: a national cross sectional study. Prev Med, v. 54, n. 2, p. 134-9, Feb. 2012. Disponível em: <http://ac.els-cdn.com/ S0091743511004828/1-s2.0-S0091743511004828-main.pdf?_tid=31322478-e6e5-11e6-a75500000aab0f02\&acdnat=1485778452_e68d16f832feffe00c150f0b60a15b8e>.

SAELENS, B. E. et al. Measuring Physical Environments of Parks and Playgrounds: EAPRS Instrument Development and Inter-Rater Reliability. Journal of Physical Activity \& Health, v. 3, p. S190-S207, 2006. Disponível em: <http://search.ebscohost.com/login.aspx?direct=true $\& \mathrm{db}=$ s3h\&AN=20971316\&lang=pt-br\&site=ehost-live $>$.

SANTOS, M. P. et al. Parental physical activity, safety perceptions and children's independent mobility. BMC Public Health, v. 13, p. 584, Jun. 15 2013. Disponível em: <https://www.ncbi. nlm.nih.gov/pmc/articles/PMC3686676/pdf/1471-2458-13-584.pdf>. 
SCHOEPPE, S. et al. Associations between children's independent mobility and physical activity. BMC Public Health, v. 14, p. 91, Jan. 29 2014. Disponível em: <https:/www.ncbi.nlm. nih.gov/pmc/articles/PMC3932047/pdf/1471-2458-14-91.pdf>.

SILVA, P.; SANTOS, M. P. Playing outdoor and practising sport: A study of physical activity levels in Portuguese children. Eur J Sport Sci, p. 1-7, Sep. 072016.

TANDON, P. et al. Physical and social home environment in relation to children's overall and home-based physical activity and sedentary time. Prev Med, v. 66, p. 39-44, Sep. 2014. Disponível em: <https://www.ncbi.nlm.nih.gov/pmc/articles/PMC4125450/pdf/nihms602463.pdf>.

TRAN, I.; CLARK, B. R.; RACETTE, S. B. Physical activity during recess outdoors and indoors among urban public school students, St. Louis, Missouri, 2010-2011. Prev Chronic Dis, v. 10, p. E196, Nov. 21 2013. Disponível em: <https://www.ncbi.nlm.nih.gov/pmc/articles/ PMC3839587/pdf/PCD-10-E196.pdf>.

VAN DEN BERG, M. et al. Visiting green space is associated with mental health and vitality: A cross-sectional study in four european cities. Health Place, v. 38, p. 8-15, Jan. 182016. Disponível em: <http://ac.els-cdn.com/S1353829216000149/1-s2.0-S1353829216000149main.pdf?_tid=42164cc6-3923-11e6-baa6-00000aacb35d\&acdnat=1466673607_53db7a6007 97eddaccca4772a2e61da2>.

WARD, J. S. et al. The impact of children's exposure to greenspace on physical activity, cognitive development, emotional wellbeing, and ability to appraise risk. Health Place, v. 40, p. 44-50, Jul. 2016. Disponível em: <http://ac.els-cdn.com/S135382921630048X/1-s2.0S135382921630048X-main.pdf?_tid=9b5592f0-d109-11e6-9cb3-00000aab0f02\&acdnat=148 3375167_7a7f1262740234654c4674421c0440ef>.

\section{Notas}

${ }^{1}$ S. M. de Oliveira contribuiu para a concepção e delineamento do estudo, coleta, análise e interpretação dos resultados, redação e revisão crítica do conteúdo intelectual. M. P. M. dos Santos participou da concepção e delineamento do estudo, além da revisão crítica do conteúdo intelectual. P. Silvia, da análise e interpretação dos resultados e revisão crítica do conteúdo intelectual. 


\section{Abstract}

Children in the parks: safety above all

Parks are outdoor environment that can contribute

to increase the children's physical activity. This study aimed to identify factors that may influence children parks use frequency of use of parks by evaluating the environmental characteristics of park and identifying factors reported by children. Descriptive and exploratory study with qualitative approach using the EAPRS tool and focal groups with children. Our results suggest that children's perceptions about park safety and maintenance can influence their park use. Transdisciplinary strategies aimed at improving safety and park maintenance are necessary to promote greater children 's park use.

> Keywords: parks; children; physical activity. 